\title{
Pelvic exenteration for colorectal cancer: oncologic outcome in 59 patients at a single institution
}

Horacio N López-Basave

Flavia Morales-Vásquez

Ángel Herrera-Gómez

Alejandro Padilla Rosciano

Abelardo Meneses-García

Juan M Ruiz-Molina

Department of Gastroenterology, Instituto Nacional de Cancerología (INCan), Mexico City, Mexico
This article was published in the following Dove Press journal:

Cancer Management and Research

10 October 2012

Number of times this article has been viewed

Background: Pelvic exenteration (PE) continues to be the only curative option in selected patients with advanced or recurrent pelvic neoplasms. A current debate exists concerning the appropriate selection of patients for PE, with the most important factor being the absence of extrapelvic disease.

Aim: To evaluate the outcome of patients submitted to exenterative surgery.

Patients and methods: A review of the clinical charts of patients with colorectal cancer who underwent PE between January 1994 and June 2010 at the Institute National of Cancerología in Mexico City was performed.

Results: We selected 59 patients, 53 of whom were females (90\%), and six of whom were males $(10 \%)$. Mean age at the time of diagnosis was 50 years (range, 21-77 years). A total of 51 patients underwent posterior PE (86\%), and eight patients underwent total PE (14\%). Operative mortality occurred in two cases (3\%), and 29 patients developed complications (49\%). Overall, 11 patients (19\%) experienced local failure with mean disease-free survival time of 10.2 months. After a mean follow-up of 28.3 months, nine patients are still alive without evidence of the disease (15\%).

Conclusions: PE should be considered in advanced colorectal cancer without extrapelvic metastatic disease. PE is accompanied by considerable morbidity (49\%) and mortality (3\%), but local control is desirable. Overall survival justifies the use of this procedure in patients with primary or recurrent locally advanced rectal cancer.

Keywords: pelvic exenteration, colorectal cancer

\section{Introduction}

Pelvic exenteration (PE) was first described by Brunschwig in 1948 as "the most radical surgical attack so far described for pelvic cancer." The overall mortality rate for the pelvic exenteration surgery was $23 \%{ }^{1}$ today, PE continues to be the only curative treatment for advanced or recurrent pelvic cancer. According to the literature, mortality rates have diminished worldwide ${ }^{2}$ due, in part, to improvements in surgical techniques and in the postoperative care of these patients. Radical exenterative surgery has mainly been used for the treatment of advanced gynecological cancer. The experience with this operation for other malignant pelvic tumors has been much more limited. The first series that described this procedure for colorectal cancer was carried out by Butcher and Spjut in $1959 .{ }^{3}$ The authors pointed out the benefits of exenterative surgery for cancer of the lower colon and realized that not every case was suitable for this procedure.

Earlier recognition of recurrent malignancies, in addition to radiotherapy and multidisciplinary care, have led to improved pelvic cancer treatment, thus avoiding
Correspondence: Horacio Noé LópezBasave

Depto de Gastroenterología, Instituto Nacional de Cancerología (INCan), Av San Fernando \#22, Col Sección XVI, Deleg Tlalpan, 14050 México, DF, México Tel +52 5556280400 ext I 20

$\mathrm{Fax}+525556280464$

Email lobohnoe@gmail.com 
the need for complete PE. ${ }^{4}$ There are a group of patients who would benefit from exenterative surgery; these individuals include those with locally advanced pelvic tumors without distant metastases, which can cause severe local problems such as pain, voiding and defecation problems, and may result in decreased quality of life.

Extensive surgery is often the only possibility for complete resection in an attempt to provide local control and palliation. In the case of involvement of the base or trigone of the bladder or prostate, total pelvic exenteration (TPE) with resection of the rectum together with the bladder, lower ureters, and internal genital organs could potentially save the patient. ${ }^{5} \mathrm{PE}$ has been well described in Mexico for the management of gynecological cancer. ${ }^{6}$

In the present study, all patients who underwent PE were reviewed. Morbidity and mortality, local disease recurrence, disease-free survival (DFS), and overall survival (OS) rates were studied; furthermore, prognostic factors for local control or survival were also analyzed.

\section{Patients and methods}

Between 1994 and 2010, 59 of 728 patients (8.1\%) with a diagnosis of either colorectal or anal cancer underwent PE at the Instituto Nacional de Cancerología in Mexico City. A review of the clinical charts of patients with this diagnosis was performed. Demographic data such as age at diagnosis, gender, and clinical status were analyzed. The tumor data gathered were size, histological type, and disease stage at the time of diagnosis, and all patients were classified according to the modified Dukes' staging system. Treatment variables analyzed included preoperative treatment, exenteration type, complete resection versus gross or microscopic positive margins, adjuvant therapy, complications, disease recurrence, and survival. For the purposes of this study, resection was defined as "complete" (disease-free margins achieved) if the entire gross tumor was removed. The "incomplete" category included patients who had microscopic or gross residual disease after surgery. Retrospective analysis of the 59 patients was performed, and follow-up data are presented as available. Statistical analysis was performed with descriptive measures.

\section{Results}

A total of 59 patients with a diagnosis of colorectal and/or anal cancer underwent PE; 53 of these patients were female $(90 \%)$ and six were male $(10 \%)$. Mean age at the time of diagnosis was 50 years (range, 21-77 years). In total, nine patients $(15 \%)$ had primary cancer recurrences. All of the patients underwent exenterative surgery; 51 patients underwent posterior pelvic exenteration (PPE) (86\%), 10 patients underwent supralevator surgery, and eight patients were submitted to TPE (14\%) (Table 1). Overall, 54 patients presented with rectal bleeding (92\%), 46 patients complained of diarrhea and/or constipation (78\%), 26 patients suffered from pain (44\%), and 23 reported weight loss (39\%).

Symptom duration ranged from 1-37 months, with an average of 10 months. In total, 31 patients (53\%) received symptomatic treatment for their pain, obstruction or bleeding; 14 of these patients ( $24 \%$ ) had undergone a nonradical surgical procedure at their local institution (Table 2). One patient received preoperative radiotherapy, and an additional five patients received both neoadjuvant chemo- and radiotherapy at our hospital. All of the patients were later submitted to exenterative surgery. Patients were analyzed preoperatively and were selected using computerized tomography and/or magnetic resonance imaging scanning. Screening for distant metastases was performed using thoracic and abdominal computerized tomography scans in all patients.

Disease-free margins were obtained in 50 patients $(85 \%)$; gross macroscopic tumor was left behind in five patients (9\%), and microscopic tumor was observed by the pathologist in four patients $(7 \%)$. In terms of mortality rates, two patients

Table I Clinical characteristics of 59 patients who underwent pelvic exenteration for colorectal cancer

\begin{tabular}{ll}
\hline General & Characteristics \\
\cline { 2 - 2 } & $\mathbf{N}$ \\
\hline Age (years) & 50 \\
Sex & \\
a. Female & 53 \\
b. Male & 6 \\
Site & \\
a. Colorectal & $58(98 \%)$ \\
b. Anal & $1(2 \%)$ \\
Histology & \\
a. Adenocarcinoma & 57 \\
b. Squamous cell carcinoma & 1 \\
c. Leiomyosarcoma & 1 \\
Tumor & \\
a. Primary & $50(85 \%)$ \\
b. Recurrent & $9(15 \%)$ \\
CEA & $13(29 \%)$ \\
Surgery types & $>10 \mathrm{ng} / \mathrm{mL}$ \\
PPE & 51 \\
$\quad$ a. PPEI & 41 \\
b. PPES & 10 \\
TPE & 8 \\
Operative time & 5 hours (4-7 hours $)$ \\
Blood loss & $900 \mathrm{~mL}(700-1500 \mathrm{~mL})$ \\
\hline Abrevions &
\end{tabular}

Abbreviations: CEA, carcinoembryonic antigen; PPE, posterior pelvic exenteration; PPES, posterior pelvic exenteration supraelevator; PPEI, posterior pelvic exenteration infraelevator; TPE, total pelvic exenteration. 
Table 2 Patients previously treated outside the institution

\begin{tabular}{llllll}
\hline & $\begin{array}{l}\text { No of } \\
\text { patients }\end{array}$ & \multicolumn{3}{c}{$\begin{array}{l}\text { Treatment at the institution } \\
\text { (prior to exenterative surgery) }\end{array}$} \\
\cline { 2 - 5 } & & Cht & Rt & Both & Surgery \\
\hline Total & 31 & & I & 5 & I \\
Surgery & 14 & & & & \\
$\begin{array}{l}\text { Hemorrhoidectomy } \\
\text { Colorectal resection }+\end{array}$ & 4 & 8 & 2 & \\
$\begin{array}{l}\text { colostomy } \\
\text { Total abdominal }\end{array}$ & 2 & I & I & \\
hysterectomy & & & & & \\
Other nonspecified & 17 & & & & \\
\hline
\end{tabular}

Abbreviations: Cht, chemotherapy; Rt, radiotherapy.

died after the procedure as a result of abdominal sepsis and pulmonary thromboembolism (3\%), and 29 patients developed complications during the postoperative period (49\%); the postoperative complications are described in Table 3. The most common intraoperative complication was major bleeding, which was observed in four patients (7\%). Other complications included wound dehiscence $(15 \%)$, abscess or wound infection (12\%), and complications associated with colostomy (10\%).

Pelvic floor reconstruction was performed in 10 patients $(17 \%)$. In eight patients, an omental flap was employed, and in the remaining two, a peritoneal flap was constructed. Mean hospital stay was 9 days (median, 10.29 days; range, 1-27 days). Seventeen patients (29\%) were taken to the intensive care unit (ICU) for immediate postoperative care. Mean ICU stay was 2 days (median, 3 days; range, 1-9 days). One patient died in the ICU.

Of the 59 patients analyzed here, 30 patients received adjuvant therapy, 16 received neoadjuvant therapy, and 13 patients were submitted to surgery alone (Table 4). Overall, 38 patients were submitted to radiation either alone or together with another chemotherapy treatment.

According to the pathology charts, the three most common types of adenocarcinomas noted among patients were

Table 3 Morbidity and mortality after exenterative surgery

\begin{tabular}{ll}
\hline Complication & No of patients (\%) \\
\hline Postoperative death & $2(3.38)$ \\
Bleeding & $4(6.78)$ \\
Pulmonary embolism & $2(3.38)$ \\
Abscess or wound infection & $7(11.86)$ \\
Evisceration & $1(1.69)$ \\
Fecal or urinary fistula & $5(8.47)$ \\
Complications of the stoma & $6(10.17)$ \\
Intestinal obstruction & $4(6.78)$ \\
Overall mortality & $2(3)$ \\
Overall morbidity & $29(49)$ \\
\hline
\end{tabular}

Table 4 Overall neo- and adjuvant treatment (survival/mortality)

\begin{tabular}{lllll}
\hline & $\begin{array}{l}\text { No neo- or } \\
\text { adjuvant }\end{array}$ & Cht adj & Rt adj & Cht-Rt adj \\
\hline Surgery & $13(6 / 7)$ & $4(0 / 4)$ & $15(8 / 7)$ & II (4/7) \\
Cht neo & 0 & 0 & 0 & 0 \\
Rt neo & $2(0 / 2)$ & $0(0 / 0)$ & $3(1 / 2)$ & $0(0 / 0)$ \\
Cht-Rt neo & $4(1 / 3)$ & $4(I / 3)$ & $1(0 / 1)$ & $2(1 / 1)$ \\
\hline
\end{tabular}

Abbreviations: Cht, chemotherapy; Rt, radiotherapy; neo, neoadjuvant; $C h t$ adj, adjuvant chemotherapy; Rt adj, adjuvant radiotherapy.

the moderately differentiated type, the well-differentiated type, and the poorly differentiated type. The moderately differentiated type was most frequently present, as it was noted among 37 patients (63\%), followed by the well-differentiated type found in 13 patients (22\%), and the poorly differentiated type occurred among seven patients (12\%). An intermediate-degree leiomyosarcoma and a moderately differentiated squamous cell carcinoma were also diagnosed (Table 1). We found only one patient was classified at Dukes' A stage (1.75\%), 18 patients at Dukes' B stage (32\%), 28 patients at Dukes' C stage (49\%), and 10 patients at Dukes' D stage (18\%); two (3\%) patients were not classified. The only patient at the Dukes' A stage never experienced tumor recurrence; conversely, three patients at Dukes'B experienced recurrence (17\%), seven patients at Dukes' C stage recurred (25\%), and one patient at Dukes' D stage experienced recurrence (10\%) (Table 5).

Overall, 37 patients died with malignancy (63\%); 11 patients (19\%) had local failure with a mean DFS time of 10.2 months. After a mean follow up period of 28.3 months, nine patients were alive without evidence of disease (15\%), two patients were still alive with tumor (3\%), and 11 patients $(19 \%)$ were lost to follow-up, and data on their disease were not collected at the last follow-up (Figure 1). A total of 46 patients received some type of oncologic treatment in addition to surgery (Figure 2).

\section{Discussion}

Pelvic exenteration plays an important role in the care of patients with advanced pelvic malignancies. Throughout 50 years of constant evolution, it has been labeled as the "gold standard" treatment for highly developed and not disseminated pelvic lesions. The procedure was first described by Alexander Brunschwig, ${ }^{1}$ but similar techniques were already being performed independently at hospital centers in North America. In the early 1940s, even prior to the actual technique being described and published worldwide, Eugene Bricker - who developed the earliest method of bladder substitution ${ }^{7}-$ believed that exenterative surgery would 
Table 5 Recurrence after exenterative surgery for colorectal and anal canal adenocarcinoma according to the Dukes' classification

\begin{tabular}{|c|c|c|c|c|c|c|}
\hline & \multicolumn{6}{|c|}{ No of patients (\%) } \\
\hline & $\mathbf{A}$ & B & C & D & NC & Total \\
\hline Total number of patients & 1 & 18 & 28 & 10 & 2 & 59 \\
\hline Local & 0 & $3(16.67)$ & $7(25)$ & $\mathrm{I}(\mathrm{I0})$ & & II (19.3) \\
\hline Latency & & 34.6 months & 8.21 months & 7.02 months & 0 & \\
\hline Distant & 0 & $6(33.33)$ & $5(17.86)$ & $5(50)$ & 0 & $16(28)$ \\
\hline Liver & 0 & I & 3 & 1 & & 5 \\
\hline Lung & 0 & 4 & 1 & 0 & & 5 \\
\hline Distant node & 0 & 1 & I & 4 & & 6 \\
\hline
\end{tabular}

Abbreviation: NC, not classified.

provide a better outcome when used for the treatment of rectal neoplasms instead of cervical cancer, due to fact that disease-free margins were obtained with greater ease.

In Mexico, the procedure is employed for the treatment of advanced pelvic malignancies, especially gynecologic tumors, which are a significant health issue in many Latin American countries. Colorectal and anal canal cancer occupies $30 \%$ of large bowel tumors ${ }^{8}$ and represents $2 \%$ of all malignancy-associated deaths in Mexico. ${ }^{9,10}$ This disease occurs in adult males beyond the sixth decade of life and presents with symptoms such as rectal bleeding and abdominal pain or discomfort.

In Mexico, PE for colorectal and anal cancer is seldom used as a means to treat a patient's targeted lesion. This is due to the fact that these lesions should fulfill certain criteria: the neoplasm should be locally advanced, and it should present without distant metastases at the time of diagnosis. Boey ${ }^{11}$ stated: "An invasive tumor, penetrating contiguous pelvic viscera but without disseminated disease, confronts the surgeon in about $6 \%$ of large bowel cancers."

Throughout history, PPE has been performed mainly to target anorectal malignancies. There is sufficient evidence to suggest that this exenteration type is usually used based on the specific organ of origin. A total of 51 patients were submitted to PPE (86.4\%), while 10 underwent supralevator surgery, and 8 patients were submitted to TPE (14\%). It is important to note that 53 patients were female $(90 \%)$;

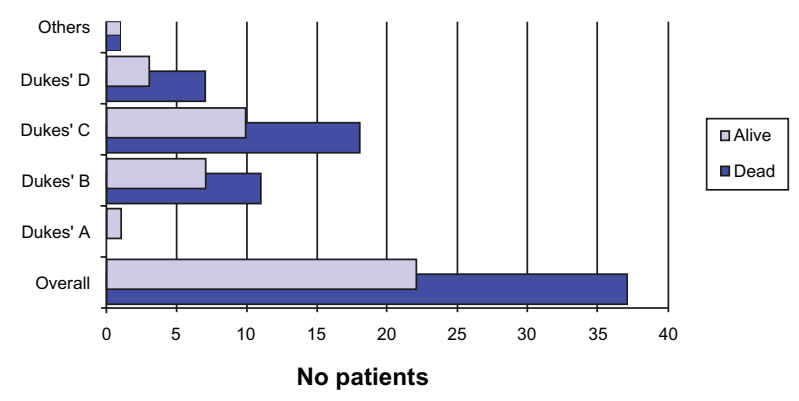

Figure I Outcome of patients according to the Dukes' classification. this is not to suggest that colorectal and anal cancer occurs more frequently in women than men, but it clarifies the idea that this tumor type involves the female uterus, vagina, and rectum, therefore creating the ideal scenario for the use of the PPE procedure.

The primary goal of exenterative surgery is to remove the central tumor mass, despite its size. Neither cure nor palliation occurs if the tumor is left behind. ${ }^{12}$ In this series, disease-free margins were achieved in 50 patients $(85 \%)$, and a remnant of the lesion was left in nine patients (15\%). Futile attempts to save an adjacent organ can result in tumor transection, and incomplete resection is generally followed by prompt recurrence and death. ${ }^{13}$

Colon and rectal surgery is prone to complications, which reflect the physiologic and anatomical nature of the systems of the particular organ involved. For years, exenterative surgery has been known to account for an important number of postoperative complications. Moreover, although significant improvements in surgical techniques and in postoperative management of the patient have occurred, potential complications after PE are numerous. Reported morbidity rates vary widely in the literature, ranging from $13 \%-77 \% .{ }^{14-17}$ On the other hand, while early studies reported operative mortality rates near $15 \%$, more recent reviews report rates closer to $3 \% ;^{18}$ this is where current improved care standards affect patient outcomes. An individual who undergoes radical pelvic surgery is at risk of early (up to 30 days after surgery)

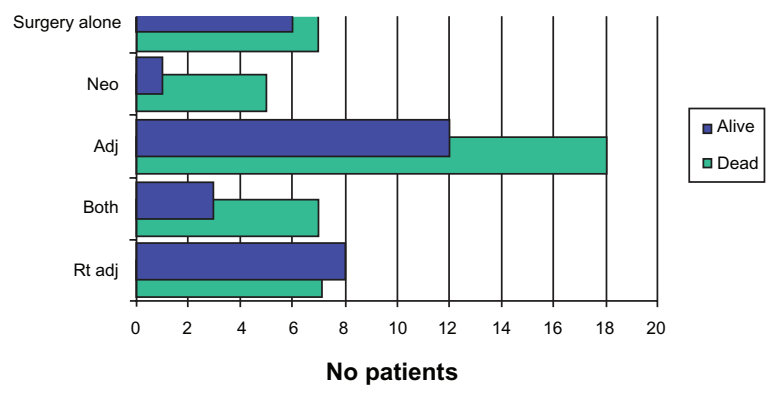

Figure 2 Outcome of patients in relation to neo- and adjuvant therapy. Abbreviation: Adj, adjuvant; Rt, radiotherapy. 
and late (later than 30 days after the surgery) complications. Our findings in this study are in line with these rates, as we obtained an overall morbidity of $49 \%$ and an overall mortality rate of $3 \%$.

Complications related to urinary diversion are most frequently cited in the literature. Our most important complications were related with stoma and wound care; this is because PPE was the procedure performed in about $81.35 \%$ of patients in the study. PPE does not require urinary diversion; as a result, the most common complications noted among patients were wound dehiscence (15\%), abscess or wound infection $(12 \%)$, and stroma complications (10\%). Some researchers have indicated that radiation therapy either preoperatively or post-procedurally is associated with higher morbidity rates, especially after the surgical event. ${ }^{16}$ Sixteen patients received radiation before the procedure, and eight of these patients submitted to radiation prior to the development of complications following radical pelvic surgery $(50 \%)$. Of the 43 patients who received no radiation therapy at all, 21 patients developed complications (49\%). We found no association between morbidity and prior radiation. There was no significant difference in the outcome of patients who underwent radiation therapy and those who did not receive such a treatment in this series; however, the effects of radiation on tissues and wound healing have been well known for years. ${ }^{18,20}$

Local tumor recurrence has been recorded in $20 \%$ to $>50 \%$ of cases in many studies. ${ }^{18-20}$ In our experience, local recurrence occurred in $19 \%$ of patients, and distant recurrence occurred in $47 \%$ of patients. Positive nodes as well as tumor size were described as the most important determinants for local recurrence of colorectal cancers that have been submitted to exenterative surgery. Risk of local recurrence has been associated with regional lymph nodes in about $20 \%-25 \%$ of cases. ${ }^{21}$ Only one patient recurred out of the twelve patients, with palpable lymph nodes noted upon clinical examination. Local recurrence remains an issue with which surgeons are required to deal in order to achieve better survival rates.

Size, as stated previously, was defined as a determinant for local recurrence of tumors, but it has been demonstrated in recent years that size does not affect recurrence or survival unless residual tumor remains after surgery. ${ }^{14}$ At our institution, many patients arrive with huge masses and highly advanced disease. Mean tumor size of patients studied here was $6.56 \mathrm{~cm}$ (range, 2-14 cm), and although the literature has overlooked the value of tumor size as a determinant for survival, a large mass implies rectal penetration by tumor, nodal spread, or direct invasion of other tissues and organs, thereby directly affecting a patient's survival rate; this is a common issue among the majority of our patients. Size, involvement of adjacent organs, previous surgery, and prior irradiation are all factors that may lead to substantial recurrence and to subsequent morbidity and mortality rates.

The primary role of radical pelvic surgery was palliation for advanced malignancies. Today, the main purpose of $\mathrm{PE}$ is to provide a cure; therefore, recurrence represents the most important failure of the procedure. Many authors have noted the absence of symptomatic pelvic recurrence even with visceral spread or carcinomatosis, such is the case in this series. ${ }^{2,18,22}$ The key item here is the gap in DFS. The Dukes' classification correlates well with the latency period and OS. In this series, patients who recurred at Dukes' B had a latency period of 34.6 months, those who recurred at Dukes' $C$ had a latency period of 8.21 months, and those who recurred at Dukes' D had a latency period of 7.02 months (Table 5). Careful patient selection is imperative in order to improve outcomes. ${ }^{22-24}$

As was the case with recurrence rates, survival is also closely related to the disease stage, but patients with nodal disease and/or systemic spread will have a poor prognosis, and exenterative surgery is palliative, not curative.

A total of 46 patients received some type of cancer treatment in addition to surgery. While we do not have significant data pertaining to this subject, we noted that the only patients that presented with a positive OS were those who submitted to radiation after the procedure (a total of eight patients survived of the 15 who received the therapy), as opposed to patients who underwent the remaining treatments. Because the percentage of local and distant recurrence remains high, we think that this factor must be included in further discussions of PE as a standard treatment for advanced pelvic malignancies.

Pelvic sarcomas are rare tumors that originate from the stroma of pelvic viscera or from the retroperitoneum. Complete resection and tumor grade are the main prognostic factors associated with increased survival rates. In the present study, only one patient with sarcoma had undergone complete tumor resection and did not experience disease recurrence in the pelvis. ${ }^{25,26}$

\section{Conclusion}

The figures for 5-year OS after exenterative surgery for colorectal cancer have not changed in recent years. The pelvis, either alone or in combination with other sites, is the earliest and most predominant site of the first tumor recurrence after adequate resection. Careful patient selection is imperative 
to improve patient outcomes. Moreover, PE should be considered in advanced colorectal cancer without extrapelvic metastatic disease. PE is accompanied by considerable morbidity (49\%) and mortality (3\%), but local control is good, which justifies PE in patients with primary or locally recurrent advanced rectal cancer.

\section{Disclosure}

The authors report no conflict of interests in this work.

\section{References}

1. Brunschwig A. Complete excision of the pelvic viscera for advanced carcinoma; a one-stage abdominoperineal operation with end colostomy and bilateral ureteral implantation into the colon above the colostomy. Cancer. 1948;1(2): 177-183.

2. Law WL, Chu KW, Choi HK. Total pelvic exenteration for locally advanced rectal cancer. J Am Coll Surg. 2000;190(1):78-83.

3. Butcher HR Jr, Spjut HJ. An evaluation of pelvic exenteration for advanced carcinoma of the lower colon. Cancer. 1959;12(4): 681-687.

4. Lopez MJ, Petros JG, Augustinos P. Development and evolution of pelvic exenteration: historical notes. Semin Surg Oncol. 1999;17(3): $147-151$.

5. Vermaas M, Ferenschild FT, Verhoef C, et al. Total pelvic exenteration for primary locally advanced and locally recurrent rectal cancer. Eur $J$ Surg Oncol. 2007;33(4):452-458.

6. López-Graniel CM, Castilleros-Pinilla C, Jiménez-Ríos MA, Munoz Gonzalez DE, Puerto Mendez V, Gonzalez EA. Exenteración pélvica en el Instituto Nacional de Cancerología. Revista del Instituto Nacional de Cancerología. 1999;45(4):230-242.

7. Bricker EM, Eiseman B. Bladder reconstruction from cecum and ascending colon following resection of pelvic viscera. Ann Surg. 1950; 132(1):77-84.

8. Office of Population Censuses and Surveys. Cancer Statistics Registrations. Series MBI, No 18. London: HMSO; 1990.

9. Garcia Carrizosa R. Incidence of gastrointestinal neoplasms in the Hospital Central Military. Prensa Med Mex. 1968;33(1):13-14.

10. Maldonado-Garza H. Cancer de colón ¿en México? [Colonic cancer in Mexico?]. Rev Gastroenterol Mex. 2001;66(3):169. Spanish.
11. Boey J, Wong J, Ong GB. Pelvic exenteration for locally advanced colorectal carcinoma. Ann Surg. 1982;195(2):513-518.

12. Jimenez RE, Shoup M, Cohen AM, Paty PB, Guillem J, Wong WD. Contemporary outcomes of total pelvic exenteration in the treatment of colorectal cancer. Dis Colon Rectum. 2003;46(12):1619-1625.

13. Appleby LH, Deddish MR. Discussion on the treatment of advanced cancer of the rectum. Proc R Soc Med. 1950;43(12):1071-1081.

14. Ledesma EJ, Bruno S, Mittelman A. Total pelvic exenteration in colorectal disease. Ann Surg. 1981;194(6):701-703.

15. Bricker EM, Modlin J. The role of pelvic evisceration in surgery. Surgery. 1951;30(1):76-94.

16. Jakowatz JG, Porudominsky D, Riihimaki DU, et al. Complications of pelvic exenteration. Arch Surg. 1985;120(11):1261-1265.

17. Eckhauser FE, Lindenauer SM, Morley GW. Pelvic exenteration for advanced rectal carcinoma. Am J Surg. 1979;138(3):412-414.

18. Vermaas M, Ferenschild FT, Verhoef C, et al. Total pelvic exenteration for primary locally advanced and locally recurrent rectal cancer. Eur $J$ Surg Oncol. 2007;33(4):452-458.

19. de Wilt JH, van Leeuwen DH, Logmans A, et al. Pelvic exenteration for primary and recurrent gynaecological malignancies. Eur J Obstet Gynecol Reprod Biol. 2007;134(2):243-248.

20. Yamada K, Ishizawa T, Niwa K, Chuman Y, Aikou T. Pelvic exenteration and sacral resection for locally advanced primary and recurrent rectal cancer. Dis Colon Rectum. 2002;45(8):1078-1084.

21. Benotti PN, Bothe A Jr, Eyer RC, Cady B, McDermott WV, Steele G. Management of recurrent pelvic tumors. Arch Surg. 1987;122(4): 457-460.

22. Williams LF Jr, Huddleston CB, Sawyers JL, Potts JR 3rd, Sharp KW, McDougal SW. Is total pelvic exenteration reasonable primary treatment for rectal carcinoma? Ann Surg. 1988;207(6):670-678.

23. Wanebo HJ, Antoniuk P, Koness RJ, et al. Pelvic resection of recurrent rectal cancer: technical considerations and outcomes. Dis Colon Rectum. 1999;42(11):1438-1448.

24. Goldberg JM, Piver MS, Hempling RE, Aiduk C, Blumenson L, Recio FO. Improvements in pelvic exenteration: factors responsible for reducing morbidity and mortality. Ann Surg Oncol. 1998;5(5): 399-406.

25. Lopes A, Poletto AH, Carvalho AL, Ribeiro EA, Granja NM, Rossi BM. Pelvic exenteration and sphincter preservation in the treatment of soft tissue sarcomas. Eur J Surg Oncol. 2004;30(9):972-975.

26. Lewis JJ, Leung D, Woodruff JM, Brennan MF. Retroperitoneal softtissue sarcoma: analysis of 500 patients treated and followed at a single institution. Ann Surg. 1998;228(3):355-365.
Cancer Management and Research

\section{Publish your work in this journal}

Cancer Management and Research is an international, peer-reviewed open access journal focusing on cancer research and the optimal use of preventative and integrated treatment interventions to achieve improved outcomes, enhanced survival and quality of life for the cancer patient. The journal welcomes original research, clinical \& epidemiological

\section{Dovepress}

studies, reviews \& evaluations, guidelines, expert opinion \& commentary, case reports \& extended reports. The manuscript management system is completely online and includes a very quick and fair peerreview system, which is all easy to use. Visit http://www.dovepress.com/ testimonials.php to read real quotes from published authors. 